\title{
DETERMINING AND SELECTING SCREEN PRINTING FORM PARAMETERS FOR PRINTING ON PAPER AND TEXTILE
}

\author{
Rozália Szentgyörgyvölgyi ${ }^{1}$ (i), Erszébet Novotny ${ }^{2}$ (D), Milán Weimert ${ }^{3}$ (iD \\ ${ }^{1}$ Óbuda University, Sándor Rejtő Faculty of Light Industry and \\ Environmental Protection Engineering, Budapest, Hungary \\ ${ }^{2}$ ANY Security Printing Company, Budapest, Hungary \\ ${ }^{3}$ Stencil Consult Bt, Budapest, Hungary
}

\begin{abstract}
The technology of screen printing is a chain of work processes which include many variants. Of all the parameters influencing the technology most occur due to the presence of screen mesh in the process. Reproduction ability doesn't simply require adherence to the technological steps but also needs a continuous creative and flexible approach and comprehensive knowledge of the entire procedure. Before preparing the printing stencil, serious planning is required; it needs to be determined during this process what stencil is needed to realize the expected quality of the print.

The goal of the examinations is to determine and choose the parameters of screen printing forms for creating the best possible quality screen printing stencils to be used with paper and textile printing, and the improvement of the quality of the screen preparation process for direct screen printing on paper and textile substrates. From the point of view of the quality of the print, the critical issues of the technology of stencil preparation is the planned thickness of the stencil, reaching the necessary Rz values, and the lighting conditions of accurate printing element reproduction. We have made prints with printing forms chosen by the parameters examined and analysed the quality of prints.
\end{abstract}

Key words: screen printing form, $\mathrm{R}_{\mathrm{z}}$, EOM, screen printing on paper and textile

\section{INTRODUCTION}

The technology of screen printing presents many challenges for the user. The most conflicting factor in the challenges of technology is probably the presence of screen mesh itself in the technology. While fulfilling its task, it often results in ragged edges of prints, blocks the flow of ink, distorts light like an optic strand, makes precise screen lighting difficult, and also might create problems with keeping the accuracy of the colour line up, since the forces affecting it during screen printing might change due to pressure and possible chemical effects of the chemical substances used. All of these can be kept under control with precise and astute planning and preparation, with the knowledge of the proper techniques, and by using the right tools and materials. The wide selection of media available also presents a challenge. An even tension of the screen mesh is an important parameter, and it must be set to be the same quality at a later time as it was before. In case of quality deviations, many problems might occur indirectly from screen tension, such as colour line up, colour correctness and size keeping. The final quality of the stencil is also affected by the continuously changing, wearing coating trough edges, and is also might affect the final result. When lighting the stencil, a weakening light source can affect the useful life of the stencil during printing, under or over tanning can harm the resolution of the print, or might push the distribution indicators of a properly set ink system to an unwanted direction. The thickness of the under tanned stencil might change due to the not polymerized and washed out developing parts, so we are once again facing a different print thickness and possibly shade of colour. In case of industrial screen printing, not only the colour correctness of the print, but also the expected resistance, the tensile strength and thermal expansion are also determined beforehand, so when preparing the stencil, we must be able to determine exactly what materials need to be used to prepare the printing stencil. The choice of textile provides the approximate thickness of the stencil, and the available resolution. The tension of the textile can be adjusted to the printing press or manual printing. The useful life of the printed image and the stencil depend on the correct selection of emulsion. It depends on the coating how thick the print is going to be, and how even the prints produced are going to be. The printing elements are created at exposure, and these must be in the correct ratio with the film positive, thus ensuring the right reproduction of the print. The developing is responsible for the clean print image (Leicester Print Workshop, 2018; Hurtz Gmbh, 2018). 
The goal of the examinations is to determine and choose the parameters of screen printing forms for creating the best possible quality screen printing media to be used with paper and textile printing.

\section{METHODS}

The elements of the screen printing form (stencil) generally examined are the thickness of the stencil, the EOM (Emulsion Over Mesh), the Rz value of the stencil (evenness of the surface of pressing side), the quality of printing edges and the quality of printing duct. Knowing these parameters helps deduce the quality of the prints, its possible faults and expected reproduction capability. The expected ink coat thickness of the print is a necessary parameter amongst the pre-determined characteristics of the print. In case of screen technology, it can be varied according to the requirements of the customer, and by choosing the thickness of the stencil within the framework of possibilities provided by the technology. In case of optimal stencil thickness it is approximately the same on the entire surface of the print, but in case of printing with a stencil which is too thick, the middle of the print is going to be thinner. Emulsion Over Mesh (EOM) shows the thickness of the stencil in $\mu \mathrm{m}$, not counting the thickness of the textile. The value represents how thick of an emulsion coat was created during coating exceeding the thickness of the textile. The effects of a too thick or a too thin EOM value can be detected on the prints as typical faults.

The $\mathrm{Rz}$ value of the stencil represents surface roughness. This value is provided in $\mu \mathrm{m}$, according to the following principles: between 3 and $12 \mu \mathrm{m}$ the Rz value is considered good. Below $3 \mu \mathrm{m}$ and above $12 \mu \mathrm{m}$ the value is not suitable. The higher the value, the more rough is the surface of the stencil, and it has a direct effect on the quality of the print. In case of a low $R_{z}$ value, ink is pressed between the print and the printing stencil at the indentations on the edges of the squeegees, and the image will have blurry contours, and will not represent clearly the image to be reproduced (Figure 1). It greatly harms the quality of the print in case of both raster prints and half-tone prints (Peyskens, 2003; Sefar Inc., 1999).

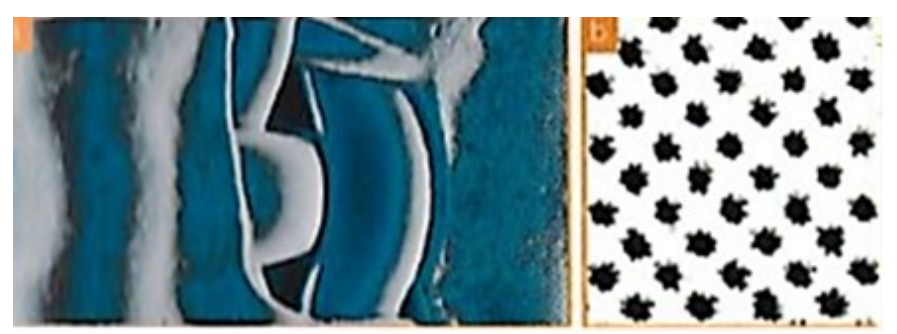

Figure 1: The effect of high Rz value on the quality of prints (Peyskens, 2003)

The determination of the quality of printing edges is done by optical examination, analysing the edges of the stencil. In case of adequate quality, the structure of the textile due to its weaving does not affect the printing edges of the stencil, and the emulsion follows the lines of the printing elements independent of the textile. The evenness of the emulsion at the edges of printing elements is called "mesh bridging". In case of optimal lighting, the points, lines and print parts of the print match the parts on the film positive exactly. In case of overexposed stencils, the size of the printing elements might differ greatly from the ones on the film positive (Figure 2), and it leads to colour deviation, incorrect spectral, point distortion, and print part loss of the print (Sefar Inc., 1999).

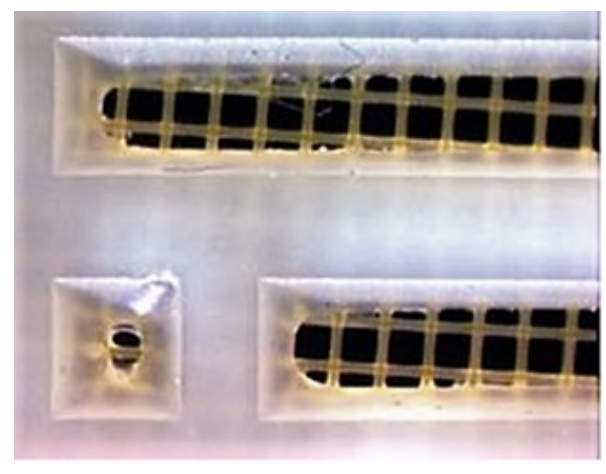

Figure 2: Closure of line in case of overexposure (Ulano, 2018) 
Significant influencing factors of print quality are also the openness of the printing duct, and therefore the quality of the squeegees created by the emulsion. In ideal case the printing duct is completely free of obstacles, therefore it does not block the flowing of ink. The squeegees are almost $90^{\circ}$ (Figure 3), so the capillary effect can help the ink to move through the stencil optimally (Sefar Inc, 1999).

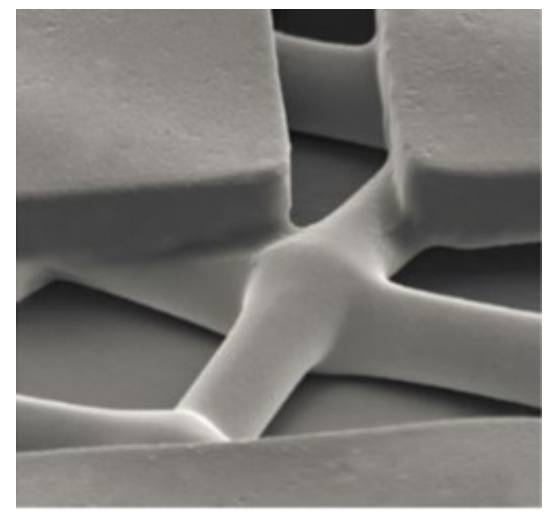

Figure 3: Squeegees of adequate quality (MacDermid Autotype Ltd., 2018)

Of all screen meshes of which screens are made of, we used SEFAR meshes made in Switzerland. These textiles have a quality of PET 1500, reduced elasticity and plasma surface treatment. The textiles are made of high viscosity polyester, have even weaving, are wearing resistant, keep their tension set at screen tensioning, are suitably elastic, and resistant to the inks and chemical substances used during printing, preparation and after treatments. Two different, simply weaved textiles were chosen, based on the planned prints and examinations; both has different weaving density (textile strand / cm). Material SEFAR PET 77.48 Y was used for textile printing, and SEFAR PET 120.34 Y material was used for paper printing (Table 1), taking the ink thickness to be reached and maximum print resolution to be reached into consideration.

Table 1: General identifying parameters of screen meshes

\begin{tabular}{|l|c|c|}
\hline \multirow{2}{*}{ Screen mesh parameter } & \multicolumn{2}{|c|}{ Screen mesh type } \\
\cline { 2 - 3 } & $77.48 \mathrm{Y}$ & $120.34 \mathrm{Y}$ \\
\hline Colour & Yellow & Yellow \\
\hline Weaving & $1-1$ & $1-1$ \\
\hline Tolerance (peace thread) & 2 & 3 \\
\hline Mesh density $($ thread$/ \mathrm{cm})$ & 77 & 120 \\
\hline Mesh-thread diameter $(\mu \mathrm{m})$ & 48 & 34 \\
\hline Mesh thickness $(\mu \mathrm{m})$ & 80 & 55 \\
\hline Tolerance $(\mu \mathrm{m})$ & 4 & 3 \\
\hline Mesh elongation $(\mu \mathrm{m})$ & 77 & 45 \\
\hline Maximum tension $\left(\mathrm{N} / \mathrm{cm}^{2}\right)$ & 35 & 28 \\
\hline
\end{tabular}

Layering takes place with a two component Diazo UV Polymer emulsion. Its medium $7000 \mathrm{mPa}$ viscosity and relatively high, $40 \%$ dry matter content provides the necessary resistance in case of both water and solvent soluble inks; at the same time adequately thick emulsion coat can be created on both types of textiles.

During the examinations the following materials were used: SEFAR 4 pneumatic Tension Device, SEFAR Tensiocheck, GRÜNIG G COAT 415 Coating Machine, GRÜNIG Coating Tub, BOCHONOW 1100 Horizontal Drying Cabinet, BOCHONOV Developing Box, KIWO ProfiWash Spray Gun, BOCHONOW 2-3-5 kW Exposure Device, BOCHONOW 2000 Manual Screen Printing Table, DEFELSKO DFT Ferro Coating Thickness Gauge, MAHR PocketSurf II Surface Roughness Device, PEAK Microscope.

The screens were layered by manual and an automatic GRÜNIG G Coat 415 machine, with a GRÜNIG coating trough with a 1.0 radius edge. The manual coating is used for preliminary testing, and machine coating is used for preparing the printing stencils, in order to have greater accuracy. The coating speed, the angular offset of the troughs, the pressure exerted by the troughs to the textile, and the emulsion distribution time before starting the pulling, in case of an automatic GrÜNIG coating machine, can be set, 
so the desired coating result can be set by parameters. When choosing the edge of the trough, a medium sharp, $1.25 \mathrm{R}$ radius trough was selected. Following the layering of emulsion, the stencils were dried in horizontal position in a BOCHONOW 1100 drying cabinet. Lighting was also taken care of by a BOCHONOW product, traditional, metal halogen bulb. After the exposure, the development of the stencil took place in a BOCHONOW washing and developing tub, completed with a KIWO Profiwash spray gun. Printing was done on a manual screen printing table, BOCHONOW 2000 Model 70/10 "A" vacuum table, with counterweight frame motion.

\section{RESULTS AND DISCUSSION}

Prior to preparing the printing stencils, examinations were carried out to determine the best technique to have the expected quality of the printing form. The following examinations were carried out:

- Examination regarding the tension keeping of the screen mesh

- Layering examinations

- Examining the effect of coating trough edge

- Examining the effect of coating speed

- Examining the effect of the amount of emulsion

- Comparing coating techniques

- Examining the effect of emulsion viscosity

- The effect of screen lighting ratio on the quality of the stencil

- Examination of prints on textile and paper media

\subsection{Examination regarding the tension keeping property of the screen mesh}

The process was started by tensioning the screen mesh on the screen frame. In order to have adequate tension and tension evenness, three tensioning techniques had been compared previously by preliminary testing; the tensioning values of a screen printing stencil tensioned with 15 minutes and 35 minutes of textile relaxation time, and one with quick, no textile relaxation time. The goal was to reach $21 \mathrm{~N} / \mathrm{cm}^{2}$ screen tension in case of the 120.34 screen mesh, and $18 \mathrm{~N} / \mathrm{cm}^{2}$ tension in case of the 77.48 screen mesh used for printing textile. The results of the tension measurements of test screens are in Table 2 . The most suitable was the result of screens made with 35 minutes of relaxation time.

Table 2: Tension keeping properties of textiles in case of various tensioning technologies

\begin{tabular}{|c|c|c|c|c|c|c|}
\hline \multirow[b]{3}{*}{ Tensioning technologies } & \multicolumn{6}{|c|}{ Tension, $\mathrm{N} / \mathrm{cm}^{2}$} \\
\hline & \multicolumn{3}{|c|}{77.48 Y screen mesh } & \multicolumn{3}{|c|}{$120.34 \mathrm{Y}$} \\
\hline & $\begin{array}{c}\text { Tension at } \\
\text { the end }\end{array}$ & 1 hour later & $\begin{array}{l}4 \text { hours } \\
\text { later }\end{array}$ & $\begin{array}{c}\text { Tension at } \\
\text { the end }\end{array}$ & 1 hour later & $\begin{array}{c}4 \text { hours } \\
\text { later }\end{array}$ \\
\hline $\begin{array}{l}\text { Quick, no textile } \\
\text { relaxation time }\end{array}$ & 20 & 18 & 16 & 22 & 22 & 22 \\
\hline $\begin{array}{l}15 \text { minutes of textile } \\
\text { relaxation time }\end{array}$ & 20 & 18 & 17 & 20 & 21 & 21 \\
\hline $\begin{array}{l}35 \text { minutes of textile } \\
\text { relaxation time }\end{array}$ & 20 & 19 & 18 & 19 & 20 & 21 \\
\hline
\end{tabular}

\subsection{Layering examinations}

In the course of testing, we compared the $R_{z}$ values and the emulsion thickness (Emulsion Over Mesh) values. Comparison and measurement of the effects of coating troughs with various radius were carried out using SEFAR PET 1500120.34 Y screen mesh, with 2/2 manual coating and Azocol Z 140 emulsion (Table 3). The examination showed that the greater the radius of the coating trough when coating the greater the EOM value. 
Table 3: The effect of various coating troughs on stencil thickness

\begin{tabular}{|c|c|}
\hline Radius of the coating trough & Stencil thickness, $\mu \mathrm{m}$ \\
\hline 0,50 & 8 \\
\hline 1,00 & 10 \\
\hline 1,25 & 12 \\
\hline
\end{tabular}

The effect of various coating speeds on the stencil thickness and their comparison regarding various SEFAR screen meshes with 2/4 layering were simulated with a Grünig G coat 415 automatic screen coating machine. The screen meshes were coated with Azocol Polyplus $S$ emulsion. The results of the examination are shown in Table 4. The examination showed that in case of the same type of textile, the slower is the coating, the thicker is the resulting emulsion coat. Another conclusion of the test is that the less dense is the screen mesh, the greater effect the speed of coating has on the attainable stencil thickness.

Table 4: The effect of various coating speeds on stencil thickness

\begin{tabular}{|c|c|c|c|c|c|c|}
\hline \multirow{2}{*}{$\begin{array}{c}\text { Screen mesh } \\
\text { type }\end{array}$} & \multicolumn{7}{|c|}{ Stencil thickness, $\mu \mathrm{m}$} \\
\cline { 2 - 7 } & 600 & 500 & 400 & 300 & 200 & 100 \\
\hline 77.48 & 28 & 30 & 32 & 34 & 35 & 37 \\
\hline 100.40 & 26 & 26 & 26 & 27 & 28 & 31 \\
\hline 120.34 & 24 & 25 & 27 & 26 & 25 & 26 \\
\hline 140.34 & 16 & 16 & 17 & 17 & 18 & 19 \\
\hline
\end{tabular}

The effect of various emulsion amounts on the stencil thickness with 2/2 layering were simulated with a Grünig G coat 415 automatic screen coating machine. The layering was done with Azocol Polyplus $\mathrm{S}$ emulsion (Table 5). The examination showed that the more emulsion is present in the coating trough, the thicker the coat will be when the coating parameters are the same. On the other hand, the larger the exposed area on the textile, the greater is the difference due to the different amount of emulsion.

Table 5: The effect of emulsion amounts on stencil thickness when the coating and emulsion is the same

\begin{tabular}{|c|c|c|}
\hline \multirow{2}{*}{ Screen mesh type } & \multicolumn{2}{|c|}{ EOM values, $\mu \mathrm{m}$} \\
\cline { 2 - 3 } & saturated in $15 \mathrm{~mm}$ high & saturated in $\mathbf{5 m}$ high \\
\hline 120.40 & 11 & 10 \\
\hline 120.37 & 21 & 17 \\
\hline 120.32 & 34 & 26 \\
\hline
\end{tabular}

The comparison of various coating techniques regarding stencil thickness were simulated with a Grünig $G$ Coat 415 automatic screen coating machine, with Azocol Polyplus S emulsion (Table 6). The result of the examination is that if the emulsion is coated in a greater number of times, the final result will be much thicker.

Table 6: Measured on "wet on wet" coating stencil/the same emulsion, coated in a different way

\begin{tabular}{|c|c|c|c|}
\hline \multirow{2}{*}{ Screen mesh type } & \multicolumn{3}{|c|}{ EOM values, $\mu \mathrm{m}$} \\
\cline { 2 - 4 } & $2 / 2$ coating & $2 / 3$ coating & $2 / 4$ coating \\
\hline 100.40 & 7 & 14 & 22 \\
\hline 120.34 & 5 & 10 & 14 \\
\hline 140.34 & 5 & 7 & 10 \\
\hline
\end{tabular}

In order to have the best Rz values, the effect of additional print side coating after the intermediate drying of the stencil was examined on a GRÜNIG G Coat 415 automatic screen coating machine, with Azocol Z 140 emulsion (Table 7). The results of the examination showed that the additional coating resulted in a minimal change of $1 \mu \mathrm{m}$ in stencil thickness. 
Table 7: Effect of additional coating stage on the thickness of stencil/ the same emulsion, coated in a different way

\begin{tabular}{|c|c|c|c|}
\hline \multirow{2}{*}{ Screen mesh type } & \multicolumn{3}{|c|}{ EOM values, $\mu \mathrm{m}$} \\
\cline { 2 - 4 } & $2 / 2+1$ coating & $2 / 3+1$ coating & $2 / 4+1$ coating \\
\hline 100.40 & 8 & 16 & 23 \\
\hline 120.34 & 6 & 11 & 15 \\
\hline 140.34 & 6 & 8 & 11 \\
\hline
\end{tabular}

The effect of differences in emulsion viscosity on stencil thickness were examined on a Grünig G Coat 415 automatic screen coating machine, with Azocol Z 140 emulsion. The results in Table 8 showed that due to the density differences in colder emulsion, stencil thickness had a higher value than the values of a similar coating done using emulsion of a higher temperature.

Table 8: The effect of emulsion temperature differences on stencil thickness, coated with the same type of emulsion of different temperature

\begin{tabular}{|c|c|c|}
\hline \multirow{2}{*}{ Screen mesh type } & \multicolumn{2}{|c|}{ EOM values, $\mu \mathrm{m}$} \\
\cline { 2 - 3 } & $15^{\circ} \mathrm{C}$ & $21^{\circ} \mathrm{C}$ \\
\hline 100.40 & 9 & 7 \\
\hline 120.34 & 6 & 5 \\
\hline 140.34 & 5,5 & 5 \\
\hline
\end{tabular}

Based on the evaluation of the results of manual coating tests, the following parameters were chosen for preparation of screens to be used for the prints:

- $\quad 77.48$ screen mesh for printing cotton textile

- $\quad 2 / 2$ emulsion coating ( $2 x$ on printing side, $2 x$ on squeegee side coating)

- emulsion coating with a coating trough with $1.25 \mathrm{R}$ radius edge

- fully filled coating trough (to $15 \mathrm{~mm}$ height)

- emulsion temperature $21 \mathrm{C}^{\circ}$ (temperature of printing room)

- $\quad$ on a Grünig G Coat 415 automatic coating machine the emulsion layering with coating speed $600 \mathrm{~cm} / \mathrm{min}$.

- 120.34 screen mesh for printing paper

- $\quad 2 / 1+1$ emulsion coating ( $2 x$ on printing side, $1 x$ on squeegee side coating, an after intermediate drying $1 x$ coating from printing side)

- emulsion coating with a coating trough with $1.25 \mathrm{R}$ radius edge

- fully filled coating trough (to $15 \mathrm{~mm}$ height)

- emulsion temperature $21 \mathrm{C}^{\circ}$ (temperature of printing room)

- $\quad$ on a Grünig G Coat 415 automatic coating machine the emulsion layering with coating speed $400 \mathrm{~cm} / \mathrm{min}$.

In the course of the examinations, our goal was to reach the desired stencil parameters, and based on our previous experience, it was easily reached with the help of a GRÜNIG G Coat 415 automatic coating machine. The parameters of the coated screens are in Table 9.

Table 9: The parameters of stencils used for final lighting and printing tests

\begin{tabular}{|c|c|c|c|c|c|c|}
\hline \multirow[b]{2}{*}{ Stencil } & \multicolumn{6}{|c|}{ Screen mesh parameters } \\
\hline & Screen mesh & $\begin{array}{c}\text { Screen } \\
\text { tension, } \\
\mathrm{N} / \mathrm{cm}^{2}\end{array}$ & $\begin{array}{l}\text { Coating } \\
\text { technology }\end{array}$ & $\begin{array}{c}\text { Stencil } \\
\text { thickness, } \\
\mu \mathrm{m}\end{array}$ & $\mathrm{EOM}, \mu \mathrm{m}$ & $\mathrm{R}_{\mathrm{z}}, \mu \mathrm{m}$ \\
\hline $\begin{array}{l}\text { Textile printing } \\
\text { stencil }\end{array}$ & $\begin{array}{c}\text { SEFAR } \\
\text { PET } 1500 \\
77.48 . Y\end{array}$ & 21 & $2 / 2+1$ & 91 & 21 & 7,7 \\
\hline $\begin{array}{l}\text { Paper printing } \\
\text { stencil }\end{array}$ & $\begin{array}{c}\text { SEFAR } \\
\text { PET } 1500 \\
120.34 . Y\end{array}$ & 21 & $2 / 1+1$ & 57 & 14 & 8,6 \\
\hline
\end{tabular}


The screens positioned as layers were dried in a BoOCHONOW 1100 horizontal dryer, on $38 \mathrm{C}^{\circ}$ temperature and 120 minutes of drying time. The temperature was checked in every 30 minutes using a mobile thermometer. The temperature fluctuation was not greater than $3 \mathrm{C}^{\circ}$, and it did not exceed the upper limit of $41 \mathrm{C}^{\circ}$. After drying, the even distribution of the emulsion coats of the screens were checked at a number of points, using a layer thickness measuring device. The results were values within the tolerance of the measuring tool $(1 \mu \mathrm{m})$, therefore we concluded that the dried emulsion coats are even, and there are no differences in thickness at the various points of the screen printing stencils.

\subsection{The effect of screen lighting ratio on the quality of the stencil}

To identify the proper lighting values, KIWO EXPOCHECK calculating film was used. By using the test film, simulating 10 different exposure times on a stencil, we were able to define the underexposure limit of the coated screen within one exposure cycle, with the help of colour change during emulsion polymerization. Overexposure limit was defined by the comparison of the size of positive and negative lines, which are the same size on the film, measured on the stencil. Developing was done with cold water and a KIWO PROFIWASH developing spray gun. The optical identification of exposure quality was carried out by the following parameters of the stencils. The colour of underpolymerized stencil is weaker, and it will be visible due to the thinning of the stencil because during developing the not fully solidified underexposed emulsion on the squeegee side will be washed out. In case of overexposure, due to point and line increase (under lighting), the positive and negative lines which are the same size on the film will close up and increase on the stencil, producing a different size. Following the identification of the correct minimal exposure value, the exposure time was defined with the help of a microscope, where the best possible resolution was found and stencil was fully tanned, taking into consideration the following: quality of printing edges, the angle of printing duct, the quality of mesh bridging, and the printing element sizes determined at the location where positive and negative lines meet (Figure 4).

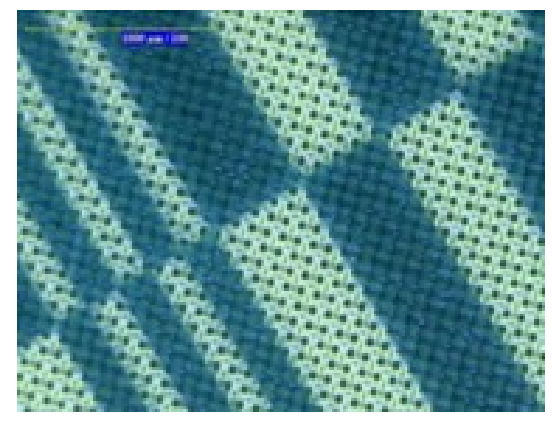

Figure 4: Size concordance of positive and negative lines

The following exposure values were detected during the examinations, which were carried out using KIWO Expocheck film, containing printing elements (Tables 10 and 11).

Table 10: The basis of exposure tests are the lighting parameters of 120.34 Y stencils

\begin{tabular}{|c|c|c|c|c|}
\hline \multirow{2}{*}{ Stencil } & \multicolumn{4}{|c|}{ Exposure time, $\mathrm{s}$} \\
\cline { 2 - 5 } & Entire exposure & Minimum exposure & Optimal exposure & Over exposure \\
\hline $\begin{array}{c}\text { Textile printing } \\
\text { stencil }\end{array}$ & 240 & 120 & 168 & 190 \\
\hline
\end{tabular}

Table 11: The basis of exposure tests are the lighting parameters of $77.48 \mathrm{Y}$ stencils

\begin{tabular}{|c|c|c|c|c|}
\hline \multirow{2}{*}{ Stencil } & \multicolumn{4}{|c|}{ Exposure time, $\mathrm{s}$} \\
\cline { 2 - 5 } & Entire exposure & Minimum exposure & Optimal exposure & Overexposure \\
\hline $\begin{array}{c}\text { Paper printing } \\
\text { stencil }\end{array}$ & 240 & 110 & 132 & 214 \\
\hline
\end{tabular}




\subsection{Examination of prints on textile and paper media}

The prepare stencil was printed on white, $100 \%$ cotton substrate, using black BOMO-Print LAC FLEX water soluble ink. The printing of the stencil for the printing of the paper was carried out using Maraster RS solvent soluble screen ink from the manufacturer MARABU. A commercial, white organic cotton T-shirt material was chosen as textile media. Art paper IGEPA CircleOffset $100 \mathrm{~g} / \mathrm{m}^{2}$ was chosen for paper media. The inspection of the quality of the print prepared with solvent based ink on paper media was done by digital microscope. The quality of the print edges, evenness and at selected locations, the correctness of the size were examined and compared to the image on the film positive to be reproduced, as shown on Figures 5 and 6.

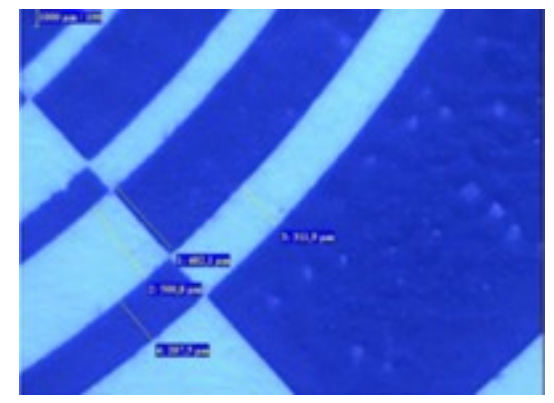

Figure 5: Size concordance of positive and negative curving lines on the print

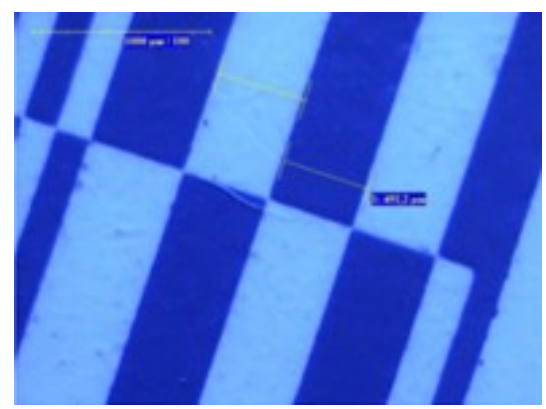

Figure 6: Size concordance of positive and negative straight lines on the print

The positive and negative lines which were to be the same size on the print showed a deviation value of less than $10 \%$. The print edges are clean, with contours, the interfering effect of the textile is minimal, barely noticeable, and there is no raggedness detected. The corners of the prints are precise, well defined, the $90^{\circ}$ angle tips are sharp, and no print loss can be seen. When comparing the raster points of the film positive and the print, the size differences on the print and film were only 1 to $3 \mu \mathrm{m}$, and it means less than $1 \%$ of deviation. The raster print shows optically acceptable image, although the edges have less contour than the line elements. The excellence of the results of the spectrophotometer measurements well represents that there is a $5 \%$ deviation in general between various shades. At the same time, the greatest difference between the values measured on the print and on the film was only $10 \%$. The results of the measurements conducted show that the printing stencil has a very good quality, and the image on the film is reproduced on the media very well. The ink distribution is minimal, the print has sharp and contour image, and sizes have only minimal deviations. Both curved and straight lines are free of raggedness and waves. Thanks to the additional printing side coating, the stencil squeegees are sharp and due to their even stencil thickness, there are no protuberances at the edge of the print. There is no ink smearing at the circular measurement elements. Due to the proper stencil thickness and thanks to the additional coating, the layer of ink created is adequate. The stencil can be printed fully, there are no areas which are not printed due to ink blockage; except for the limit due to physical limits originating from the screen mesh and the emulsion resolution capability, which defines an approximately $120 \mu \mathrm{m}$ size as the lower printable limit.

The inspection of the quality of print on cotton textile is done by checking the contours of the image, the line size retention at printing, the possible print distortions between the edges of the print, the sharpness 
of lines, the coating property of the print, and the bleeding of the print edges. Measurement points were chosen on the film used for printing on cotton media: a circular, a line, and a fine resolution text area.

The coating property of the black print is very strong despite the absorbing white textile substrate; when stretching the media, it does not crack or break, and keeps its colour. The print edges have contour, the distribution of ink is average, and there is no strong bleeding along the textile strands. It can be observed very well at the nonprinting element print which is about $100 \mu \mathrm{m}$ thick and located at the edge of the circular printing element; it did not slur on the print (Figure 7).

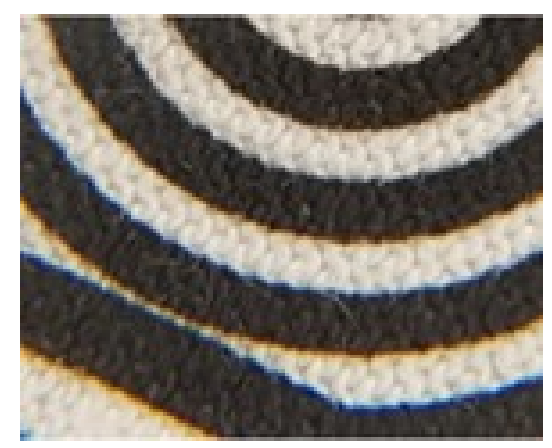

Figure 7: Checking the position of ink blurring on the print

We have measured the $1 \mathrm{~mm}$ line thickness ensured by the film at the line print with the help of a measuring field magnifier (Figure 8). Taking into consideration the stretching capability of the textile, the size of the print did not deviate significantly from the size expected. The lines show sharp contours, and thanks to the sharp print edges, the ink has almost three dimensional effect despite the minimal coat thickness.

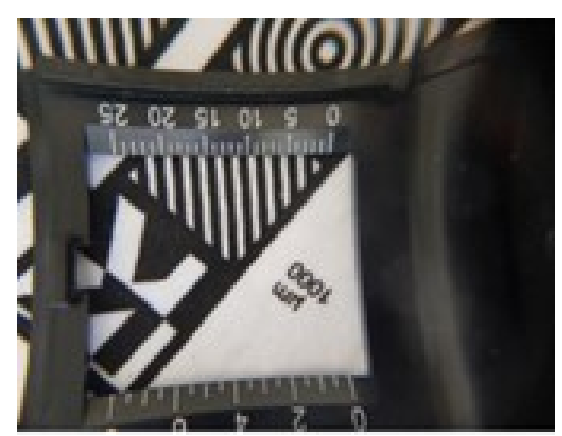

Figure 8: Checking the size of lines on the print

All characters were fully printed in the fine, small text area; the printing elements have contour and are well defined. The resulting stencil is of good quality, its coat thickness is optimal, and its Rz value is excellent despite the fact that the substrate of the stencil is a 77.48, medium "homespun" weaved screen mesh. The edges of the stencil are straight, the textile does not decrease the resolution capability of the emulsion. Due to adequate exposure, the squeegees are almost at a right angle, the stencil is developed to the necessary rate and without remnants, thus ensuring an adequate printing duct for the screen ink flow.

\section{CONCLUSIONS}

The goal of this work is to perfect the preparation of a screen printing stencil, and the improvement of the quality of the screen preparation process for direct screen printing on paper and textile substrates. The right stencil was chosen by examinations. From the point of view of the quality of the print, the critical issues of the technology of stencil preparation is the planned thickness of the stencil (EOM), reaching the necessary Rz values, and the lighting conditions of accurate printing element reproduction. We have made prints with printing forms based on the parameters examined, and analysed the quality of prints. The measurements conducted show that the printing stencils were of very good quality. 
Measurements conducted with the help of a microscope showed that on average, the print image on the film was reproduced on art paper with less than $5 \%$ deviation. The examination of the textile print was carried out with less precise measurements, but with inspections fitting to the base characteristics of the media. Excellent stencil and print have resulted from the effect of even and straight edges of the print contours, the size keeping of the lines, the non-distorted and not blurred full image, and the ink with excellent covering capabilities.

\section{REFERENCES}

[1] Hurtz Gmbh: "Screen printing frames" URL: https://www.hurtz.de/en/products/frames/screenprinting-frames (last request: 2018-09-12).

[2] Leicester Print Workshop: "A brief history of screenprinting", URL: http://www.leicesterprintworkshop.com/printmaking/screenprinting/a_brief_history_of_ screenprinting/ (last request: 2018-09-12).

[3] MacDermid Autotype Ltd.: "Anleitung - Belichtungsgeräte", URL: http://autotype.macdermid.com/how-to-guides/details/206/anleitung-belichtungsgerate (last request: 2018-09-20).

[4] Peyskens, A.M.: "Szitakészítés Műszaki Alapelvek", (SaatiPrint S.p.A., Budapest, 2003).

[5] Sefar Inc.: "Handbook for screen printers", (Sefar Printing Division, Thal, 1999.)

[6] ULANO: "Frequently Asked Questions" URL: http://www.ulano.com/FAQ/FAQexposure.htm (last request: 2018-09-21).

(C) 2018 Authors. Published by the University of Novi Sad, Faculty of Technical Sciences, Department of Graphic Engineering and Design. This article is an open access article distributed under the terms and conditions of the Creative Commons Attribution license 3.0 Serbia (http://creativecommons.org/licenses/by/3.0/rs/). 